\title{
The Impact of Meaningful Game Narratives on Attitudes towards Racial Outgroups
}

\author{
Munifa Shaza \\ Nanyang Technological \\ University \\ munifash001@e.ntu.edu.sg
}

\author{
Valerie Yu \\ Nanyang Technological \\ University \\ valeriej001@e.ntu.edu.sg
}

\author{
Katrina Alvarez \\ Nanyang Technological \\ University \\ alva0002@e.ntu.edu.sg
}

\author{
Vivian Hsueh Hua Chen \\ Nanyang Technological \\ University \\ chenhh@ntu.edu.sg
}

\begin{abstract}
Serious games with interactive narratives have been studied for their potential to influence emotions, behaviors and attitudes concerning real-world people and issues. Past research suggests that the meaningfulness of a narrative is potentially determined by the level of consequence following choices within the narrative. These choices may be the key to improving the effectiveness of an interactive narrative for prosocial outcomes such as improving perceptions towards racial outgroup members. This study examined how consequential and inconsequential choices in meaningful game narratives influence prosocial outcomes towards racial outgroups, as well as the level of meaningfulness perceived by players. Participants in the pre-post experimental study played a newly developed serious game and generally showed improved perceptions towards racial others after gameplay. However, there were no significant differences with regards to prosocial outcomes and perceived meaningfulness of the game narrative between consequential and inconsequential choice conditions of the game.
\end{abstract}

Keywords: interactivity, consequences, video games, meaningful choices, attitudinal change

\section{Introduction}

Interactive media such as video games, which allow users to control actions and modify experiences, might be more effective than non-interactive media in inducing participants' prosocial attitudinal change towards social issues and outgroup members $[1,5]$. Serious games are those that are designed with a purpose beyond entertainment [1]. Various studies have found serious games to be effective in achieving positive health and educational goals [2, 3]. Additionally, serious games have also been used as a medium to spread social awareness and train interpersonal skills [4]. While society has long struggled with racist attitudes, these have risen sharply amid the COVID-19 pandemic [6]. It is relevant and timely to investigate the potential of using video games to improve perceptions towards outgroups delineated by racial and ethnic differences.

Interactive games create a more direct, self-related connection between player and game world events [7] as they engage and involve players directly in the content of a game [8], enabling players to create stories personalized to their own values [9]. Research suggests that interactive narratives may influence subsequent attitudes and emotions concerning realworld people and issues [10], as people typically adopt attitudes that align with the choices they make to avoid cognitive dissonance [11]. Gentile et al. [12], found that players assigned to play prosocial games that encouraged helping behaviours (Chibi Robo and Super Mario Sunshine) subsequently also displayed helpful behaviours. Peng et al. [1] showed that playing an interactive narrative game about a humanitarian crisis in war-torn Darfur, Darfur is Dying, resulted in significant improved prosocial attitudes towards the humanitarian issue highlighted in the interactive game, compared with participants who had only read a text version of the game's narrative or watched a playthrough of Darfur is Dying.

However, a series of studies on the impact of a persuasive digital game, Against All Odds, on willingness to help refugees did not find evidence that playing a persuasive game could have more effective prosocial results than watching a video or reading a printed text [13]. The inconclusive findings about the effect of serious games on attitudes towards outgroups may be due to the different narrative structures and choices games offer and the impact they have on players. As games also provide opportunities for players to experience narratives where they can actively make decisions that affect the story, this creates a greater sense of engagement with the 
storyline and connection to in-game characters [14]. Therefore, this study explores how players perceive meaningfulness of game narrative and how their choices within meaningful game narratives influence their perceptions and prosocial intentions towards racial outgroup members.

\section{Theoretical Background}

\subsection{Choices in Meaningful Narratives}

Within the context of serious games, narratives are usually designed to be meaningful, which is defined as imparting purposeful learning and relevance to the players [15]. Choices in these games provide players with information to make decisions that may affect the outcome of a game [16] and enable players to feel more connected to the story through their own actions [17]. They typically push the player to tackle complex issues such as moral dilemmas and conflicts between values [18]. For choices to be effective in inducing prosocial tendencies, players should perceive them as meaningful [19] within the context of the narrative. To be meaningful is to be thought-provoking and moving [14].

Example studies that used meaningful narratives suggest that choices that impact the course of a game provide players some control over its outcomes, enabling the learning of prosocial and moral behaviour to take place [20-21]. In one study, participants exhibited more positive attitudes towards the homeless after playing a game where the choices made as a homeless person impacted their survival on limited means, compared with participants who had read a non-interactive first-person account on being homeless and were not required to make any decisions pertaining to the homeless, or as a homeless individual [22].

A study on the serious game Migrate, where players made crucial in-game choices as a Mexican immigrant attempting to illegally cross the border into the United States, found more positive effects regarding Mexicans in the US afterwards [5]. This suggests that choices available to players in meaningful game narratives allow players to reflect on their decisions and that the subsequent consequences of those choices may be effective in promoting attitude change.

H1: A game narrative with choices will positively change players' attitudes (H1a), stereotypes (H1b), and empathic response (H1c) towards racial outgroups.
$\mathrm{H} 2$ : A game narrative with choices will increase players' prosocial intentions towards racial outgroups.

\subsection{Meaningfulness and Consequences}

There are a variety of game choices in any given game. We want to further understand how the nature of game choices influences perceptions and prosocial intentions towards racial outgroup members.

Past research has found that consequences are one way for players to perceive meaningfulness in an interactive narrative. Steinemann et al. [19] suggested that the number and severity of consequences following in-game decisions determine the level of meaning that players perceive, with more consequential outcomes resulting in more perceived meaning. Elson et al. [9] have also argued that consequences and outcomes in games equip players with the ability to alter the story to fit what they personally perceive to be as relevant or meaningful.

On the other hand, Nay and Zagal [16] proposed that even inconsequential choices may be able to induce meaning by helping the player reflect on the subtleties and motives behind their actions without a focus on the outcomes. This shifts the focus instead to the characters that players control, with players defining the morality of their characters for themselves, and according to their own personal ethical perspectives.

Through the performance of a player's personal perspectives, the storyline deviates to one that is more personally relevant to the player, rather than simply adhering to in-game ethical perspectives that predetermine whether a player receives a "bad ending" or a "good ending" — a difference players are often occupied with [16].

Given these opposite arguments, it is still uncertain whether the consequences of in-game choices, defined by degree, has an impact on the perceived meaningfulness of the game and players' perceptions and prosocial intentions.

Hence, the following research questions are asked:

RQ1: Is there a difference between the consequential and inconsequential choices regarding perceived meaningfulness of the game narrative?

RQ2: Is there a difference in players' attitudes, stereotypes, and empathic response towards racial outgroup members between the consequential and inconsequential game narrative choices?

RQ3: Is there a difference in players' prosocial intentions towards racial outgroup members between the consequential and inconsequential game narrative choices?

RQ4: What is the effect of consequential choices on changes in players' attitudes, stereotypes, empathic 
response, and prosocial intentions towards racial outgroups?

RQ5: What is the effect of inconsequential choices on changes in players' attitudes, stereotypes, empathic response, and prosocial intentions towards racial outgroups?

\subsection{Appreciation}

The concept of meaningfulness is often operationalized and measured as appreciation, a state of gratification [23], and describes media experiences of being moved, of gaining insight into important and difficult questions on life's purpose, and of connecting with others [24]. Appreciation is highly relevant in this study because as expanded on in Rogers et al. [24], and Iten et al. [18], while meaningful game experiences can be related to the depth of story and its characters, the experiences which most distinguished meaningful from fun experiences were those that involved moral choices and dilemmas.

\section{Methods}

We designed an online experiment to test how the meaningfulness in game narrative influences perception towards racial outgroup members and if there is a difference in changes of perception for racial outgroups between consequential and inconsequential choices conditions.

\subsection{Participants}

Seventy participants (39 females, 31 males) were recruited by email using snowball sampling. Participants were between the ages of 21 and 46, and the average age was 23.34 (SD = 3.10). Each participant received a digital voucher worth 10 Singapore Dollars as an incentive.

\subsection{Procedures}

After recruitment, all participants received an email containing the link to the online questionnaire, where the stimulus game was embedded. Participants were given a brief text introduction to the game's story, describing a supernatural threat to the playercharacter's town, rival clans, the conflict between them, the player-character's clan membership, and the player-character's role in defeating the threat. Pregame questions then measured their initial perceptions towards racial outgroup members and demographic information.
Upon completion of the pre-test questions, participants were randomly assigned to either the consequential or inconsequential choice condition. Thirty-five participants were assigned to each condition based on the rule of thumb of 30 suggested under the Central Limit Theorem [29]. Each experimental group was provided with different narrative primes before gameplay.

Players in the consequential condition were given these instructions:

You will be playing as the heir of the Ice Gatekeeper clan. Keep in mind that the choices you make in the game may have severe consequences on the game's events.

Players in the inconsequential condition were given these instructions:

You will be playing as the heir of the Ice Gatekeeper clan.

Participants were automatically directed to play the video game in their assigned conditions. After finishing the game, they completed a post-test questionnaire that measured their post-game perceptions and prosocial intentions towards racially outgroup members and levels of appreciation of the game narrative.

\subsection{Stimulus}

The stimulus used was a web game developed in Unity and embedded in Qualtrics. The narrative of the game follows a player character who is forced to work alongside an unliked outgroup character of a different race in order to save their people from a crisis. Two versions of the game were created, one for each choice condition.

The player character protagonist is a high-status hero type in the game's society and belongs to the powerful Ice Gatekeeper clan. The crisis threatening the town forces them to work with the main NPC named Zork, a mage from the Ramu clan who live as outcasts. Together, the player and Zork resolve the different challenges that eventually end the crisis. Throughout the game, players are given choices that define their relationship to Zork as friendly or hostile. These choices are inconsequential in that they do not affect the direction and linearity of the game except for slight differences in text dialogue.

The same choices exist in both the inconsequential and consequential conditions, except for one additional choice in the consequential condition. In the consequential condition, players must make an additional choice between (1) saving Zork from potential death or injury or (2) following their duty to save their own threatened ingroup townspeople. Specifically, if the player choses to save Zork from 
potential death or injury, the player loses the one item in their possession - an enchanted knife - that would aid in the resolution of the crisis, resulting in an ending where the fate of the town remains ambiguous. However, the Ice Gatekeeper clan and Ramu people will reconcile amicably. On the other hand, if the player choses to leave Zork behind and follow their duty to their clan, they save their town from crisis. They will lose the companionship and help of Zork for the remainder of the game up until the ending. When Zork does return at the very end of the game, he is injured, and the state of relations between the two clans is left antagonistic. Players are briefly informed by a pre-decision monologue that the choice picked would have consequences for the game's outcome, so that players are aware that the choice is a consequential one. With this moral dilemma, complexity is added to the narrative, with long-term consequences for the characters and the outcome of the story.

\subsection{Measures}

The pre-test questionnaire included the measures for attitudes, stereotypes and empathic response towards outgroup members, prosocial intentions and demographic information. The same measures were used in the post-test, with the addition of a scale measuring for appreciation of game narrative. Participants were asked to rate "people of a different race or ethnicity from your own" as outgroup members.

3.4.1. Attitudes Towards Outgroup Members. Participant's attitudes towards outgroup members were measured via a feeling thermometer scale [25] for warmth, The 3 items included "Cold / Warm", "Positive / Negative", and "Favorable / Not Favorable". They were rated between 0 (negative feeling) to 100 (positive feeling). The feeling thermometer had a pre-test Cronbach's $\alpha$ of .895 and a post-test $\alpha$ of .846 .

3.4.2. Stereotypes Towards Outgroup Members. To measure stereotypes towards outgroup members, a 7point semantic differential scale on 5 adjective pairs (e.g., Good/Bad, Pleasant/Unpleasant, Honest/Dishonest) was adapted from Osgood et al [26]. The semantic differential scale had a pre-test Cronbach's $\alpha$ of .947 and a post-test $\alpha$ of .944 .

3.4.3. Empathic Response. Four adjectives (sympathetic, warm, compassionate, moved) from the Emotional Response Questionnaire [27] that are typically identified as a measure of empathy [30] were used in the questionnaire. In the instructions, participants were asked to reflect on the experiences of racial outgroup members or minorities in their community to guide their answers. They responses were averaged to provide empathy scores. Cronbach's $\alpha$ was .873 for the pre-test and .943 for the post-test scale.

3.4.4. Prosocial Intentions. A 4-item prosocial intentions scale adapted from Pavey et al. [28] was also included to see if there could be any effect on prosocial outcomes beyond improving attitudes. For example, participants were asked on their likelihood to go out of their way to help a friend in need from a racial outgroup or give up their time to do something that would benefit the racial outgroup. These intentions were measured using a 7-point Likert scale $(I=$ not at all likely, $7=$ extremely likely $)$. Average pre- and post-test scores were calculated for each participant. Cronbach's $\alpha$ was .709 for the pre-test and .871 for the post-test scale.

3.4.5. Appreciation. The Appreciation Scale developed by Oliver and Bartsch [23] was adapted and modified slightly to fit the context of the study. This consisted of 3 items, ("I found the story of this game to be very meaningful", "I was moved by the story of this game", and "The story of this game was thoughtprovoking"), measured on a 7-point Likert scale ( $1=$ strongly disagree, $7=$ strongly agree $)$. These 3 items were computed to give an average score for appreciation. Cronbach's $\alpha$ was 879 .

\subsection{Data Analysis}

Prior to statistical tests, we ran normality tests to check assumptions. For not normally distributed variables, we used non-parametric tests where appropriate. To investigate changes in pre-test to posttest scores, we conducted paired sample t-tests and sign tests as appropriate to investigate the differences in each condition. We ran follow-up independentsamples t-tests and Mann-Whitney $U$ tests to determine whether these differences were significant between conditions. This was done separately for (1) attitudes, (2) stereotypes, (3) empathy, (4) willingness to help (prosocial intentions), and (5) appreciation.

A significance level of $p=.05$ was used to investigate the potential relationships framed in our research questions. 


\section{Results}

\subsection{Descriptive Statistics and Tests of Normality}

Descriptive statistics for pre- and post-test reported attitudes, stereotypes, empathy, prosocial intention and appreciation, for each experimental group, and can be found in Table 1 .

Table 1. Descriptive statistics for consequential and inconsequential conditions

\begin{tabular}{|c|c|c|c|c|c|c|c|c|}
\hline & \multicolumn{4}{|c|}{ Consequential condition } & \multicolumn{4}{|c|}{ Inconsequential condition } \\
\hline & \multicolumn{2}{|c|}{ Pretest } & \multicolumn{2}{|c|}{ Posttest } & \multicolumn{2}{|c|}{ Pretest } & \multicolumn{2}{|c|}{ Posttest } \\
\hline & $\mathrm{M}$ & SD & $\mathrm{M}$ & SD & $\mathrm{M}$ & SD & M & SD \\
\hline Attitude & 53.66 & 18.14 & 63.46 & 20.83 & 55.45 & 19.16 & 67.62 & 18.08 \\
\hline Stereotypes & 5.12 & 1.09 & 5.28 & 1 & 5.11 & 1.04 & 5.46 & 0.9 \\
\hline Empathy & 4.68 & 0.92 & 4.74 & 1.17 & 4.51 & 1.11 & 4.87 & 1.17 \\
\hline Prosocial Intentions & 5.66 & 0.76 & 5.51 & 1.18 & 5.51 & 0.81 & 5.56 & 0.97 \\
\hline Appreciation & - & - & 4.66 & 1.12 & - & - & 4.77 & 1.36 \\
\hline
\end{tabular}

\subsection{Effects of Game choices on Attitudes Towards Outgroup}

After removing outliers, the distribution of scores in the inconsequential condition was not normal. Nonparametric tests were thus used to detect attitude change. Specifically, a Wilcoxon signed-rank test was run to determine if there was a statistically significant improvement in attitude scores before $(M d n=50.00)$ and after game play for all participants $(M d n=66.17)$, $z=4.163, p<.001$. This suggests support for H1a, that the game narrative would improve players' attitudes towards racial outgroups.

An exact sign test was conducted for both the consequential and inconsequential conditions to determine the effect of playing the game on attitudes toward outgroup members. In the consequential condition, there was a statistically significant median increase in attitude $(M d n=1.667)$ between the pre-test $(M d n=50.0)$ and post-test scores $(M d n=65.667), z=$ $2.457, p=.015$. For participants in the inconsequential condition, there was also a significant median increase $(M d n=5.0)$ in attitudes towards outgroup members from pre-test $(M d n=53.0)$ to post-test scores $(M d n=$ $66.667), z=2.874, p=.004$. This partially answers RQ4 and RQ5 by showing that offering both consequential and inconsequential game choices can improve these attitudes.

A Mann-Whitney $U$ test was run to determine if there were differences in the attitude score change between the two conditions after playing the game.
Distributions of the attitude score changes for both groups were similar, and median attitude score change was not statistically significantly different between the consequential $(M d n=1.67)$ and non-consequential ( $M d n=5.00)$ condition, $U=664, z=.61, p=.54$. This suggests that there is no difference between groups in players' attitudes towards racial outgroups, partially answering RQ2.

\subsection{Semantic Differentials Relating to Stereotypes}

Similarly, the distribution of scores for stereotypes by participants in both conditions was not normal. A Wilcoxon signed-rank test was run. There was a statistically significant improvement in semantic differential scores before all participants played the game $(M d n=5.00)$ compared to after they played the game ( $M d n=5.40), z=3.12, p=.002$. This supports H1b.

Non-parametric sign tests showed no significant median change in stereotyping $(M d n=0)$ between pretest $(M d n=5.0)$ and post-test scores $(M d n=5.20), p$ $=0.143$, for those in the consequential condition. This partially answers RQ4 by showing that having consequential choices had no effect on players' stereotypes. On the other hand, participants in the inconsequential condition showed a significant median change in stereotypes $(M d n=0)$ between pretest $(M d n=5.20)$ and post-test scores $(M d n=5.60, p$ $=.007)$. The increase reflects an inclination away from negatively stereotyping the outgroup, suggesting that having inconsequential choices improves these perceptions, partially answering RQ5. 
A Mann-Whitney $U$ test was run to determine if there were differences in the semantic differential change between the two conditions after playing the game. Distributions of the semantic differential changes for both groups were similar, and median semantic differential change was not statistically significantly different between the consequential $(M d n$ $=0.00)$ and non-consequential $(M d n=0.00)$ condition, $U=675.5, z=0.81, p=.42$. This partially answers RQ2.

\subsection{Empathic Response}

Scores for empathic response were not normally distributed, even after removing outliers among participants in the inconsequential condition. A Wilcoxon signed-rank test was run to determine if there was a statistically significant improvement in empathic response scores before all participants played the game $(M d n=4.75)$ compared to after they played the game $(M d n=5.00), z=2.39, p=.017$. H1c was supported.

Exact sign tests showed no significant median change in empathic response $(M d n=0)$ between the pre-test $(M d n=4.5)$ and post-test scores $(M d n=5.0)$, $p=0.52$, for participants in the consequential condition. This suggests no effect of consequential choice on empathic response (RQ4). For those in the inconsequential condition, however, there was a significant increase in empathic response $(M d n=0.25)$ between pre-test $(M d n=4.75)$ and post-test $(M d n=$ $5.0), z=2.514, p=.012$. This suggests that playing with inconsequential choice had a positive effect on players' empathic response (RQ5).

A Mann-Whitney $U$ test was run to determine if there were differences in the empathic response change between the two conditions after playing the game. Distributions of the empathic response changes for both groups were similar, and median empathic response change was not statistically significantly different between the consequential $(M d n=0.00)$ and non-consequential $(M d n=0.50)$ condition, $U=726, z$ $=1.35, p=.18$. This partially answers RQ2, with no difference in impact on empathic response.

\subsection{Prosocial Intentions}

After removing two major outliers in each condition, the distribution of scores for prosocial intentions was normal. Paired samples $t$-tests were run to determine whether prosocial intentions changed from pre- to post-test. There was no significant change from pre- $(M=5.54, S D=1.07)$ to post-test $(M=5.58$, $S D=0.78), t(69)=-0.38, S E=0.11, p=.706$ scores for all participants, failing to support $\mathrm{H} 2$.

For participants in the consequential condition, there was no significant change between pre-test $(M=$ $5.659, S D=0.78)$ and post-test scores $(M=5.735, S D$ $=0.747)$. Participants in the inconsequential condition, on the other hand, showed improved prosocial intentions from pre-test $(M=5.523, S D=0.832)$ to post-test $(M=5.735, S D=0.684), M=0.212(S E=$ $0.091), t(32)=2.328, p=.026, d=0.41$. Cohen's $d$ suggests a small positive effect size. These results suggest no effect of consequential choice (RQ4) and positive effect of inconsequential choice (RQ5) on players' prosocial intentions.

An independent-samples t-test was run to determine if there were differences in prosocial intention changes between the two conditions. Prosocial intention change was lower in the consequential condition $(x=0.08, S D=0.54)$ than the inconsequential condition $(x=0.21, \mathrm{SD}=0.52)$, however it was not a statistically significant difference, $M=-0.14,(S E=0.13), t(64)=-1.04, p=$ .30 , to answer RQ3.

\subsection{Appreciation}

Two outliers were removed from the consequential group for analysis. Appreciation scores for both conditions were then found normally distributed according to Shapiro-Wilk's test (consequential, $p=$ .378; inconsequential, $p=.231$ ). An independent samples $t$-test was then run, revealing no significant difference in appreciation scores $(M=-0.33, t(66)=$ $1.26, p=.21, d=.31$ ) between the two conditions. This suggests no difference between having consequential or inconsequential choices; participants in both conditions perceived the game as meaningful (RQ1). 


\section{Discussion}

The results suggest that playing a prosocial interactive narrative game with choices can improve attitudes, stereotypes, and empathic responses towards racial outgroups. This was observed in both the consequential and inconsequential versions of the game, providing some evidence that interactive narrative need not be consequential to improve perceptions. This is consistent with the finding on appreciation.

Previous literature has suggested that narratives shaped by player actions are more likely to produce meaningful experiences that they can relate to [9]. Based on the appreciation results, players from both conditions reported that they found the game narrative meaningful. The lack of difference might suggest that simply having the illusion or perception of choice is enough for players to find meaning in a game.

One source of meaning might have been identification with the player-character, which can facilitate changes in players' attitudes based on their understanding of game events [10]. Another source of meaning might have come from engagement with the plot, where the conflict between the fictional races was revealed to be a misunderstanding due to lost history. Players might have found the experience of uncovering this truth while working with the outcast character Zork to reach the protagonist's goal to be meaningful.

It was intended that, by facilitating this meaningful experience, the game would facilitate improvement of perceptions towards racial outgroups, in line with past work where serious games have improved attitudes towards homeless individuals [22].

However, players' prosocial intentions did not increase. Other factors such as direct, consistent and stable exposure to the attitude object (in this case, a prosocial game) over time [32] and strength of the attitudinal change felt by the participant [33] may be required to promote improved behavioral intentions. Rusch [34] has also suggested that attitudinal change is more attainable than behavioral change, because although attitudinal change can provide powerful suggestion, any subsequent behavioral change that is not accompanied by a change of identity only increases the chances of reverting to previous norms or status quo.

Players in both conditions showed improved attitude towards racial outgroups. Only players in the inconsequential condition had improved scores in stereotyping, empathy, and prosocial intentions. This may be because inconsequential choices encourage players to reflect on the characters' morality as it shifts the focus of players' reflection from attaining the optimal outcome or ending in gameplay to a consideration of moral positions [16].

One limitation of the study is that the design of the consequential and inconsequential conditions could be further improved. In the game, the differences between the consequential and inconsequential conditions were narrative priming and a single choice with actual consequences for the outcome of the game. It may be useful to further investigate the impact of consequential and inconsequential choices in future studies by manipulating factors such as the number and severity of consequences.

Another limitation and future research direction is that participants' in-game choices to define their relationship with Zork as either friendly or hostile were not examined in the current study, as the focus was only on whether consequence or lack of consequence had an impact on post-game attitudes. This is a promising direction for future study in relation to the potential of inconsequential choices to promote desired outcomes.

Last in future research direction is whether an inverse effect is possible. In other words, whether a deliberate manipulation of players' perceptions, such that they believe their actions have little or no consequence, continues to promote reflection and attitude change.

\section{Conclusion}

This study showed that playing a meaningful narrative prosocial game improved attitudes and empathy and reduced stereotypes towards racial outgroup members, but not their intentions to help. Specifically, players in the inconsequential choice condition of the game showed improved attitude, empathy and prosocial intentions and reduced stereotypes towards racial outgroups. There is no difference found regarding all the dependent variables between the consequential and inconsequential choices conditions.

\section{Acknowledgements}

We wish to acknowledge the funding support for this project from Nanyang Technological University under the Undergraduate Research Experience on CAmpus (URECA) programme. This work was supported by the Singapore Ministry of Education under Grant MOE2017-T2-2-145. 


\section{References}

[1] W. Peng, M. Lee, and C. Heeter, "The Effects of a Serious Game on Role-Taking and Willingness to Help.", Journal of Communication 60 no. 4, 2010, pp. 723-742.

[2] C.M. Jones, L. Scholes, D. Johnson, M. Katsikitis, and M.C. Carras, "Gaming well: Links between videogames and flourishing mental health.", Frontiers in Psychology 5, 2014, pp. 1-8.

[3] B. Harrington, and M. O'Connell, "Video games as virtual teachers: Prosocial video game use by children and adolescents from different socioeconomic groups is associated with increased empathy and prosocial behaviour.", Computers in Human Behavior 63, 2016, pp. 650-658.

[4] G. Pereira, B. António, P. Rui, A. Paiva, F. Bellotti, M. Kravcik, and R. Klamma, "Serious Games for Personal and Social Learning \& Ethics: Status and Trends.", Procedia Computer Science 15, 2012, pp. 53-65.

[5] S. Parrott, F.R.D. Carpentier, and C.T. Northup, "A Test of Interactive Narrative as a Tool Against Prejudice.", Howard Journal of Communications 28, no. 4, 2017, pp. 374-389.

[6] C. Yong, 2020. "Growing wave of racism and hate crimes as coronavirus spreads." Straits Times, 04//. https://www.straitstimes.com/world/unitedstates/growing-wave-of-racism-and-hate-crimes-asvirus-spreads.

[7] C. Klimmt,Serious games and social change: Why they (should) work, Routledge, 2009

[8] G.H. Iten, S.T. Steinemann, and K. Opwis,"Choosing to help monsters: A mixed-method examination of meaningful choices in narrative-rich games and interactive narratives.", Conference on Human Factors in Computing Systems - Proceedings 2018, 2018, pp. 1-13.

[9] M. Elson, B. Johannes, J.D. Ivory, and T. Quandt, "More than stories with buttons: Narrative, mechanics, and context as determinants of player experience in digital games.", Journal of Communication 64 , no. 3 , 2014, pp. 521-542.

[10] M.C. Green, and K. M. Jenkins, "Interactive narratives: Processes and outcomes in user-directed stories.", Journal of Communication 64, no. 3, 2014, pp. 479500.

[11] J. Cooper, and R. H. Fazio, "A New Look at Dissonance Theory." , Advances in Experimental Social Psychology, edited by L. Berkowitz, Academic Press, 1984, pp. 229-266.

[12] D.A. Gentile, C. A. Anderson, S. Yukawa, N. Ihori, M. Saleem, K.M Lim, A. Shibuya, A.K. Liau, A. Khoo, B.J. Bushman, L.R Huesmann, and A.Sakamoto,"The effects of prosocial video games on prosocial behaviors: International evidence from correlational, longitudinal, and experimental studies.", Personality and Social Psychology Bulletin 35, no. 6), 2009, pp. 752-763.

[13] J. Van 'T Riet, A.C. Meeuwes, L. Van Der Voorden, and J. Jansz, "Investigating the Effects of a
Persuasive Digital Game on Immersion, Identification, and Willingness to Help.", Basic and Applied Social Psychology 40, no.4, 2018, pp. 180194.

[14] M.B. Oliver, N.D. Bowman, J. K. Woolley, R. Rogers, B. I. Sherrick, and M.-Y Chung. "Video games as meaningful entertainment experiences.", Psychology of Popular Media Culture 5, no. 4, 2016, pp.390-405.

[15] E. Naul, M. Liu. "Why Story Matters: A Review of Narrative in Serious Games.", Journal of Educational Computing Research 58, no. 3, 2019, pp.687-707.

[16] J.L. Nay, and J.P. Zagal, "Meaning without consequence: Virtue ethics and inconsequential choices in games.", ACM International Conference Proceeding Series Part F1301 (August 2017), 2017

[17] B. Bostan, Ö. Yönet, and V. Sevdimaliyev, 2020. "Empathy and Choice in Story Driven Games: A Case Study of Telltale Games.", 2020, pp. 361-378.

[18] G.H. Iten, K. Opwis, and S.T. Steinemann,"To save or to sacrifice? - Understanding meaningful choices in games.", CHI PLAY 2017 Extended Abstracts Extended Abstracts Publication of the Annual Symposium on Computer-Human Interaction in Play (October), 2017, pp. 495-502.

[19] S.T. Steinemann, G. H. Iten, K. Opwis, S. F. Forde, L. Frasseck, and E. D. Mekler,"Interactive Narratives Affecting Social Change.", Journal of Media Psychology 29, no. 1, 2017, pp. 54-66.

[20] L. Vikaros, and D. Degand, "Moral development through social narratives and game design.", In Ethics and game design: Teaching values through play, IGI Global, 2010, pp. 197-215.

[21] A. Bandura, W.H. Freeman, and R. Lightsey, "Selfefficacy: The exercise of control.", Springer, 1999, pp. 158-166.

[22] D. Ruggiero, "The effect of a persuasive social impact game on affective learning and attitude.", Computers in Human Behavior 45, 2015, pp. 213-221.

[23] M.B. Oliver, and A. Bartsch, "Appreciation as audience response: Exploring entertainment gratifications beyond hedonism.", Human Communication Research 36, no. 1, 2010, pp. 53-81.

[24] R. Rogers, J. Woolley, B. Sherrick, N.D. Bowman, and M.B. Oliver, "Fun versus meaningful video game experiences: A qualitative analysis of user responses.", The Computer Games Journal 6, no. 1-2, 2017, pp. 63-79.

[25] D. Zavala-Rojas, Thermometer Scale (Feeling Thermometer). In Michalos A.C. (eds) Encyclopedia of Quality of Life and Well-Being Research, Springer, Dordrecht. 2014.

[26] C.E. Osgood, G.J. Suci, and P.H. Tannenbaum. The measurement of meaning, Oxford, England: Univer. Illinois Press. 1957.

[27] J.S. Coke, C.D. Batson, and K. McDavis, "Empathic mediation of helping: a two-stage model.", Journal of Personality and Social Psychology 36, no. 7, 1978, p. 752.

[28] L. Pavey, T. Greitemeyer, and P. Sparks, "Highlighting relatedness promotes prosocial motives and 
behavior.", Personality and Social Psychology Bulletin 37, no. 7, 2011, pp. 905-917.

[29] C.J. Anderson, Central limit theorem, In I.B. Weiner and W.E. Craighead (Eds.), The Corsini Encyclopedia of Psychology. Wiley, 2010.

[30] D.C. Batson, M.P. Polycarpou, E. Harmon-Jones, H.J. Imhoff, E.C. Mitchener, L.L. Bednar, T.R. Klein, and L.Highberger, "Empathy and attitudes: Can feeling for a member of a stigmatized group improve feelings toward the group?.", Journal of Personality and Social Psychology 72, no. 1, 1997, p. 105.

[31] S.T. Steinemann, G. H. Iten, K. Opwis, S. F. Forde, L. Frasseck, and E. D. Mekler,"Interactive Narratives Affecting Social Change.", Journal of Media Psychology 29, no. 1, 2017, pp. 54-66.

[32] L.R. Glasman, and D. Albarracin, "Forming attitudes that predict future behavior: A meta-analysis of the attitude-behavior relation.", Psychological bulletin 132, no. 5, 2006, p. 778 .

[33] A. Brügger, and B. Höchli, "The Role of Attitude Strength in Behavioral Spillover: Attitude MattersBut Not Necessarily as a Moderator.", Frontiers in Psychology 10, 2019.

[34] D.C. Rusch, Making deep games: Designing games with meaning and purpose, CRC Press, 2017. 